\title{
Ultrasound simulation technique as state-of-health estimation method of lithium-ion batteries
}

\author{
$1^{\text {st }}$ J.P. Gaviria-Cardona \\ Nanotechnology engineering faculty \\ Universidad Pontificia Bolivariana \\ Medellin, Colombia \\ https://orcid.org/0000-0002-0650-8740
}

\author{
$4^{\text {th }}$ Whady Florez-Escobar \\ Mechanical engineering faculty \\ Universidad Pontificia Bolivariana \\ Medellin, Colombia \\ https://orcid.org/0000-0003-3977-0371
}

\author{
$2^{\text {nd }}$ Michael Guzman-De Las Salas \\ Mechanical engineering faculty \\ Universidad Pontificia Bolivariana \\ Medellin, Colombia \\ https://orcid.org/0000-0002-8304-8864
}

\author{
$5^{\text {th }}$ Raul Valencia-Cardona \\ Nanotechnology engineering faculty \\ Universidad Pontificia Bolivariana \\ Medellin, Colombia \\ https://orcid.org/0000-0002-3763-3034
}

\author{
$3^{\text {rd }}$ Nicolas Montoya-Escobar \\ Nanotechnology engineering faculty \\ Universidad Pontificia Bolivariana \\ Medellin, Colombia \\ https://orcid.org/0000-0002-1251-1356
}

\author{
$6^{\text {th }}$ Hader Vladimir Martinez \\ Mechanical engineering faculty \\ Universidad Pontificia Bolivariana \\ Medellin, Colombia \\ https://orcid.org/0000-0002-4693-2466
}

\begin{abstract}
Ultrasound is a non-destructive technique recently proposed to estimate Lithium-ion batteries degradation. However, recent research has been devoted towards understanding the physical phenomena behind the ultrasonic wave propagation through a Lithium-ion battery. To achieve this, the secondorder-scalar elastic and acoustic wave equations are solved with explicit and implicit finite difference method, considering the interfaces between materials with different physical properties. Results showed that implicit method presents less noise than the explicit scheme. In addition, changes in the physical properties of battery materials that occur in charge and discharge processes, highly affect the ultrasonic wave propagation inside the battery. Finally, this study demonstrates the feasibility of using numerical methods as a precursor of battery degradation estimator.
\end{abstract}

Index Terms-Lithium-ion batteries, Ultrasound, State-ofHealth, finite-difference-method, non-destructive estimation

\section{INTRODUCTION}

Sales of electric vehicles (EVs) have been growing all over the world and considering that the batteries of these systems are in some cases still being changed at $70-80 \%$ of their nominal capacity [1], the use of second-life batteries (SLBs) is creating an environment conducive to a circular economy (new) market in the world [2]. In addition, from the point of view of the use of components and materials, it is also the promoter of Recycled batteries (RBs). However, it is to be expected that in the next decade the return flows of SLBs and RBs, will have an increasing commercial interest as the lack of sources of critical materials, especially cobalt, nickel, and manganese will become increasingly evident. As a preliminary fact, cathode active material accounts for more than $20 \%$ of the overall cost of current lithium-ion (Li-ion) battery technologies based on NMC chemistry. For the same case, other raw materials may account for more than $50 \%$ of the cost associated with the cathode. Thus, from the perspective of a circular economy and considering what would be the use of

This work was supported by the Royal Academy of Engineering in the United Kingdom
SLBs and RBs, the future scenario for the most representative electric vehicle segments and energy storage market, would also mean energy savings, GHG reduction, and lower water consumption. The Li-ion batteries are attractive because its high power, energy densities, and long cycle life [3]. However, they are complex systems prone to premature unexpected failure, and a quantitative evaluation of battery health can help to predict catastrophic failures that may lead to fire or explosion [4]. Thus, it is important to monitor the batteries critical internal states, which include the degradation level, also called "State of Health" (SoH) [5].

During electrical cycling and aging, the modulus and density distribution of the battery changes. Thus, different methods (i.e., Coulombic or impedance method) have been proposed to monitor physical material variations related to the electrochemical battery processes. However, these are time consuming and cannot be applied in real time. For that reason, ultrasonic measurements are recently proposed as a non-destructive technique to evaluate the $\mathrm{SoH}$, in order to identify changes in the internal battery materials [3] [4] [5]. Nonetheless, it is difficult to deduce from the pattern obtained in the ultrasound, the relationship between battery materials and the ultrasonic wave propagation [6].

Efforts to understand the ultrasonic wave propagation in a battery have been tackled in different papers. Numerically, the wave equation used in this particular problem seems to be very scarce in the specialized literature. For example, Davies [6] simulated the propagation of the ultrasonic signal in a charged and a discharged battery, considering the different values of density and elastic modulus in both states. More recently, Copley et al. [7] studied the effects that battery and pulsing parameters have and how these alter the nature of a wave. Moreover, they faced strategies to deal with the diverse nature of battery cell geometries. However, they did not focused in the numerical method accuracy, as they used the explicit Finite Difference Method (FDM), numerical technique that has been 
reported to present truncation error [8]. Furthermore, they did not considered the electrolyte (ELT) interface that is present in liquid state.

Thus, the aim of this study is to understand the physical phenomena underlying the propagation of an ultrasonic wave through a Li-ion battery. To achieve this, the elastic wave equation is solved within a domain with solid materials. Furthermore, as an initial approach, a locked ideal-fluid formulation is applied to the wave equation in order to consider the liquid phase conformed by the ELT. Therefore, boundary and interface conditions must be considered. To find the numerical solution of the wave equation, an explicit and implicit FDM are implemented and compared. Finally, an interface analysis is carried out between the battery materials to show how the wave propagation is affected by the physical properties.

\section{PRoblem Formulation}

A Li-Ion battery is mainly conformed by foils that contain a cathode, anode and separator as solid phase, permeated with an ELT [13]. In the ultrasound technique, the transducer emits an enveloped sinusoidal compressional pulse through the lithiumion cell that consists of different thin layers. The propagation velocity and the wave amplitude in each material depends on the physical material properties (density, elastic and bulk modulus). When the wave strikes an interface, the wave is reflected and transmitted. Thus, a wave can be sensed in two layers: Where the transducer is placed and the opposite layer. Hence, in this paper, we study the changes in wave propagation for a given material, using reported values for the mechanical properties for the cathode, anode and ELT materials.

\section{A. Governing Equations}

As mentioned before, a wave propagates through the Liion battery materials. Since these components are solids (i.e., anode, cathode, and separator) and liquids (i.e., ELT), different mathematical models are required for each state to describe the interaction between the ultrasonic wave and the specific material. Thus, for the solid phase and the liquid phase, an elastic and acoustic wave model are briefly described respectively.

1) Elastic wave equation: The derivation of the secondorder-scalar elastic wave equation is given by a balance between dynamics forces and forces by the stress. The first term is derived from the second Newton's law in terms of a differential volume with dimensions $d x d y d z$ and density $\rho$ :

$$
d F=(\rho d x d y d z) \frac{\partial^{2} \xi}{\partial t^{2}}
$$

Where $\xi$ is the deformation. In the other hand, for the forces generated by an stress $\sigma$ over an area $A$, the static force equation is $F=\sigma A$. If a differential element is considered, the material suffers a force by an stress in $x$ and $x+d x$, both with the same magnitude but opposite directions. This is called the net stress $\Delta \sigma$. Hence, the differential force equation due to stresses is

$$
d F=\frac{\partial \sigma}{\partial x} d x d y d z
$$

Then, the balance between the dynamic forces (1) and the forces by the pressure (2), the continuum equation can be obtained

$$
\frac{\partial \sigma}{\partial x}=\rho \frac{\partial^{2} \xi}{\partial t^{2}}
$$

Finally, after applying the relationship between the deformation $\xi$ and the stress $\sigma$ through the strain $\epsilon$, and considering the elastic term of the Voigt model, the stress can be written in terms of the strain as

$$
\sigma=\gamma \epsilon=\gamma \frac{\partial \xi}{\partial x}
$$

where $\gamma$ is the Young's modulus. Replacing (4) in (3), the second-order-scalar elastic wave equation is obtained

$$
\frac{\partial^{2} \xi}{\partial t^{2}}=\frac{1}{c^{2}} \frac{\partial^{2} \xi}{\partial x^{2}}
$$

With $c^{2}$ as the squared velocity and it is defined as $c^{2}=\frac{\gamma}{\rho}$

2) Acoustic wave equation: Considering that the ELT inside the Li-ion batteries represents a small layer in the system, this phase can be described as an ideal locked fluid inside a cylinder shape volume. The wave propagation through a fluid is the difference between the initial density $\rho_{0}$ and initial pressure $P_{o}$ and final $\rho$ and $P$. These kind of waves are known as acoustic density or density wave, and acoustic pressure or pressure wave, as follows

$$
\Delta \rho=-\rho_{0} \frac{\partial \xi}{\partial x}, \Delta P=-B \frac{\partial \xi}{\partial x}
$$

where $B$ is the Bulk modulus. Then, similarly as in the solid case, applying a balance between the forces by the movement and the forces by the pressure $\sum F=m a=P A$, the following equation can be derived

$$
\frac{\partial P}{\partial x}=-\rho_{0} \frac{\partial^{2} \xi}{\partial t^{2}}
$$

Replacing the pressure wave when $\Delta P \rightarrow 0$, and applying the derivative of $P$ respect $x$, the acoustic wave equation for an ideal fluid can be obtained

$$
\frac{\partial^{2} \xi}{\partial t^{2}}=\frac{1}{c^{2}} \frac{\partial^{2} \xi}{\partial x^{2}}
$$

where $c^{2}$ is the wave squared velocity and it is the relation between the Bulk modulus and the density $c^{2}=\frac{B}{\rho_{0}}$. Note that the viscous component for the liquid has not been considered as this formulation represents an ideal fluid [9]. However, for a more accurate description, this term should be taken.

In spite of the similarities between (3) and (8), it is important to note that are analogous equations and represent different phenomenon. While (3) describes a wave traveling through a solid material, (8) does it for a fluid.

\section{B. Boundary conditions}

1) Left boundary: To simulate the wave emitted by the transducer, a Dirichlet boundary condition is applied with a cosine function that travels towards the internal domain:

$$
\xi(x, t)=A \cos \left(\frac{-2 \pi t}{T}\right) .
$$


where $A$ is the amplitude. Afterwards, the input is removed, and at the first node $(i=0)$, the deformation takes the following Dirichlet condition, known as fixed boundary

$$
\xi_{0}^{j}=\xi(0, j)=0 .
$$

Finally, a Neumann boundary condition is applied in order to add a reflecting condition at the boundary.

$$
\xi_{0}^{j}=\frac{\partial \xi(0, j)}{\partial x}=0
$$

2) Interface: As mentioned before, the domain consists of folded materials with different physical properties. However, the second order wave equation presents a constant wave velocity. Thus, an interface boundary condition is required. For this, a match between the deformation at the node that connect two materials must be considered, as follows:

$$
\left[\gamma \frac{\partial \xi}{\partial x}\right]_{L}=\left[\gamma \frac{\partial \xi}{\partial x}\right]_{R}
$$

3) Right Boundary conditions: For all the cases, the same Neumann boundary condition (11) is applied.

\section{NUMERICAL SOLUTION}

For this study, FDM is used as a numerical method. This is done by converting PDEs into a linear algebraic equation through a discretization process. In this paper, the explicit and implicit discretization of (5) and (8) were used to solve the wave equation, in order to compare which method is more suitable for this problem. The resulting algebraic equation used in the implicit method (three central point) and explicit method (five central point) for internal nodes are

$$
\begin{gathered}
-\lambda \xi_{i-1}^{j+1}+(1+2 \lambda) \xi_{i}^{j+1}-\lambda \xi_{i+1}^{j+1}-2 \xi_{i}^{j}+\xi_{i}^{j-1}=0 \\
\xi_{i}^{j+1}=\Delta t^{2} c^{2} \frac{\left(\xi_{i+1}^{j}-2 \xi_{i}^{j}+\xi_{i-1}^{j}\right)}{\Delta x^{2}}+2 \xi_{i}^{j}-\xi_{i}^{j-1}
\end{gathered}
$$

respectively, where $\lambda$ is

$$
\lambda=\frac{\gamma \Delta t^{2}}{\rho \Delta x^{2}}
$$

\section{NUMERICAL RESULTS}

As mentioned before, the studies found in the specialized literature for this particular problem have employed the explicit FDM. However, papers had proved that the explicit FDM presents truncation errors that are not negligible and using other finite difference scheme might result in a more accurate solution [8]. This can be seen in figure 1, where the approximate solution of the wave equation obtained by the implicit method, was compared with the explicit scheme for a particular time. The result shows that by proportionally increasing the number of nodes in the domain, the implicit method display a smoother curve whereas the explicit scheme still revealing noise. For that reason, the explicit method requires the compliance of the Courant convergence condition to have a more accurate solution and it can be corrected by decreasing steps in time and space. Noise can also be diminished by increasing points in the discretization formulation, however, this implies a greater computational time. In the other hand, implicit FDM is unconditionally stable, so a larger increment steps in time can be employed. Therefore, implicit FDM might be more suitable for this particular problem rather than the explicit FDM, due to the approximation accuracy, if the truncation error present in the explicit scheme is not corrected.

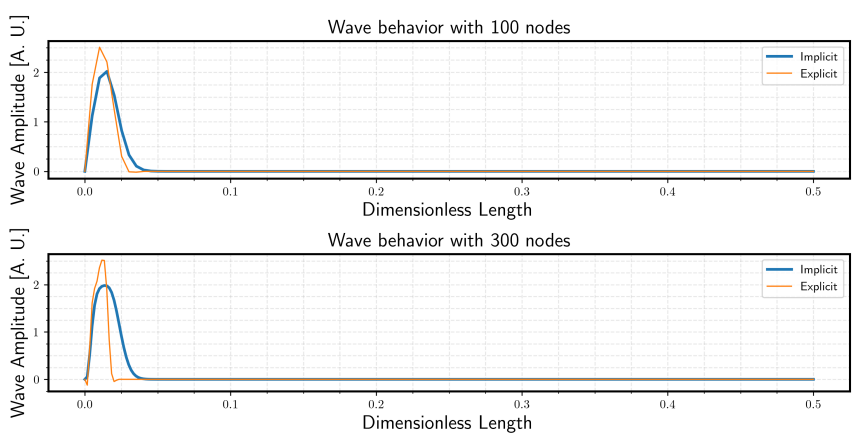

Fig. 1. Explicit and implicit FDM comparison

To explore the phenomena behind the wave propagation through finely structures, a simple layer system was conducted. Here, a non-dimensionalization is performed in the spatial axis. It can be proved that this process does not affect the wave propagation if the material properties are hold constant.

\begin{tabular}{|c|c|c|}
\hline \multirow[t]{2}{*}{ Material } & \multicolumn{2}{|c|}{ Properties } \\
\hline & Density $\left(\mathrm{Kgm}^{-3}\right)$ & $\boldsymbol{K} \vee \boldsymbol{G}(P a)^{a}$ \\
\hline Anode charged & 1934 & $1.19 \times 10^{10}$ \\
\hline Anode discharged & 1954 & $4.32 \times 10^{9}$ \\
\hline Cathode charged & 3408 & $1.16 \times 10^{10}$ \\
\hline Cathode discharged & 3408 & $1.25 \times 10^{10}$ \\
\hline Benzene ELT & 877 & $1.05 \times 10^{9}$ \\
\hline CTC ELT $^{b}$ & 1594 & $1.32 \times 10^{9}$ \\
\hline
\end{tabular}
Furthermore, the material properties are taken from experimental and computed values reported in the literature [6], and are shown in Table I.

TABLE I

BATTERY MATERIAL PROPERTIES

${ }^{\mathrm{a}} G$ is the elastic modulus and $K$ the bulk modulus. ${ }^{\mathrm{b}}$ Carbon tetrachloride

In figure 2, a solid-solid interface is simulated for three different times in a charged and discharged battery, in order to identify how battery materials affect the wave propagation. It can be seen from the result that after $0.899 \mu \mathrm{s}$, the wave is near to strike the right boundary in the upper case, whereas in the discharged battery, the wave is still traveling through the third material. This results evidence that the wave propagates faster in the charged battery than in the discharged one. The explanation relies in the internal battery effects during cycling and discharging processes. In the charging process the lithium ions are taken apart from the transition metal that conform the cathode, and move to the anode where they are stored between the graphite basal planes. During this process, the lithium electrons travel through an external circuit and are kept 
in a copper collector. In the discharging process, the lithium ions return to the transition metal through the electrolyte and separator. Thus, when the Lithium ions are inside the anode (charged battery), its volume increases in a higher proportion than the mass. Thus, considering the proportion between $\rho \propto V^{-1}$, and $c^{2} \propto \rho^{-1}$, the wave velocity increases as Li-ion battery is more charged $c^{2} \propto V$. Mukhopadhyay et al. [13], suggested that these structural changes due to Li-ion incorporation in graphite, is the increased interlayer spacing of the basal planes. Also, they found that the elastic modulus is a key property that affects the wave propagation, and found that lithiation increases the modulus perpendicular to the graphite basal planes (by $150 \%$ upon full lithiation).

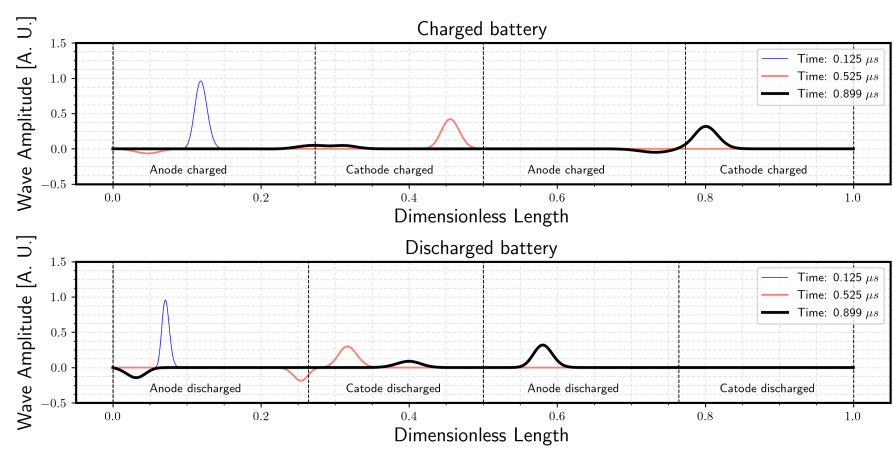

Fig. 2. Wave propagation through a charged and discharged battery

The solid-liquid interface is presented in the Figure 3, where the ELT is placed between the anode and cathode phases. Here, in the same way of the result shown above, the CTC based ELT shows higher density, resulting in a decrease in the wave velocity. Furthermore, as a validation tool, the reflected $A_{r}$ and transmitted wave amplitude $A_{t}$ at each interface are verified in function of the material acoustic impedance $Z$.

$$
A_{r}=\frac{Z_{1}-Z_{2}}{Z_{1}+Z_{2}} A_{i}, A_{t}=\frac{2 Z_{1}}{Z_{1}+Z_{2}} A_{i}
$$

With $A_{i}$ as incident amplitude. From the last equation, when the wave travels from the anode to the ELT, $A_{r}$ and $A_{t}$ are positive amplitudes for both materials. Contrarily, when the wave moves from the ELT to the cathode, $A_{r}$ and $A_{t}$ takes a negative and positive amplitude value, respectively. This behaviour can be seen in figure 3 , where at $0.525 \mu \mathrm{s}$, the wave just passed through the interface, both $A_{r}$ and $A_{t}$ result in a positive value. Subsequently, when the wave moves from the ELT to the cathode, $A_{r}$ is negative and $A_{t}$ positive. The same behaviour can be seen in the figure 2 and this phenomena is known as mode conversion [6].

\section{CONCLUSION}

Using FDM's to study an ultrasonic wave that travels through a battery proves that simulation can be a useful tool for providing data to make correlations between Li-ion battery degradation and ultrasound measurements. Also, it allows to understand the relationship between the wave propagation and the internal battery materials in different charged battery
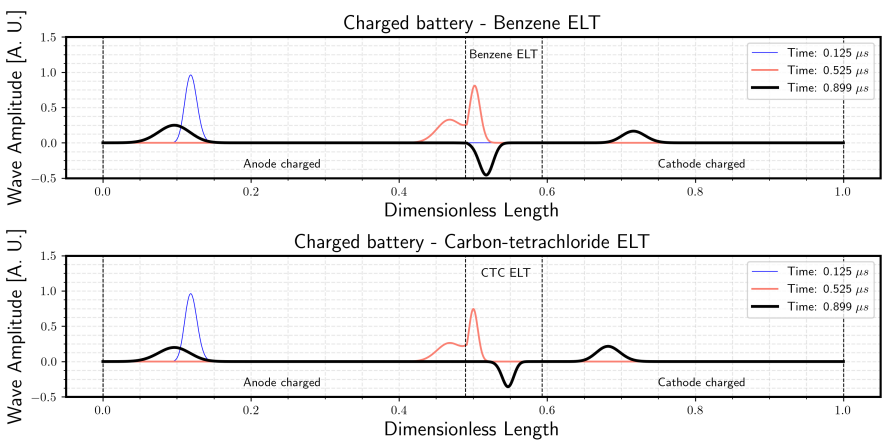

Fig. 3. Liquid-solid interface

levels, providing information about the internal state of Li-ion batteries.

\section{REFERENCES}

[1] S. I. Sun, A. J. Chipper, M. Kiaee, and R. G. A. Wills, "Effects of market dynamics on the time-evolving price of second-life electric vehicle batteries," vol. 19, no. December 2017, pp. 41-51, 2018 DOI:10.1016/j.est.2018.06.012.

[2] M. Anna, F. Guarino, S. Longo, M. Ferraro, and M. Cellura, "Energy and environmental benefits of circular economy strategies: The case study of reusing used batteries from electric vehicles," J. Energy Storage, vol. 25, no. April, p. 100845, 2019 DOI:10.1016/j.est.2019.100845.

[3] J. B. Robinson, M. Pham, M. D. R. Kok, T. M. M. Heenan, D. J. L. Brett, and P. R. Shearing, "Examining the Cycling Behaviour of LiIon Batteries Using Ultrasonic Time-of-Flight Measurements," J. Power Sources, vol. 444, no. July, p. 227318, 2019.

[4] J. Tian, R. Xiong, and W. Shen, "A review on state of health estimation for lithium ion batteries in photovoltaic systems," eTransportation, vol. 2, p. 100028, 2019.

[5] P. Ladpli, F. Kopsaftopoulos, and F. K. Chang, "Estimating state of charge and health of lithium-ion batteries with guided waves using built-in piezoelectric sensors/actuators," J. Power Sources, vol. 384, no. February, pp. 342-354, 2018.

[6] G. Davies, "Characterization of Batteries Using Ultrasound: Applications for Battery Management and Structural Determination," ProQuest Diss. Theses, no. June, p. 206, 2018.

[7] R. Copley, D. Cumming, Y. Wu and R. Dwyer-Joyce, "Measurements and modelling of the response of an ultrasonic pulse to a lithium-ion battery as a precursor for state of charge estimation", Journal of Energy Storage, vol. 36, p. 102406, 2021. Available: 10.1016/j.est.2021.102406

[8] B. Ataie-Ashtiani and S. Hosseini, "Numerical errors of explicit finite difference approximation for two-dimensional solute transport equation with linear sorption", Environmental Modelling \& Software, vol. 20, no. 7, pp. 817-826, 2005. Available: 10.1016/j.envsoft.2004.04.010

[9] J. E. Greenspon, "Acoustics, Linear," Encycl. Phys. Sci. Technol., pp. 129-167, 2003, doi: 10.1016/b0-12-227410-5/00009-0.

[10] M. Kimble and R. White, "A five-point finite difference method for solving parabolic partial differential equations," Computers \& Chemical Engineering, vol. 14, no. 8, pp. 921-924, 1990.

[11] M. Kumar, "A three-point finite difference method for a class of singular two-point boundary value problems," Journal of Computational and Applied Mathematics, vol. 145, no. 1, pp. 89-97, 2002.

[12] E. Sokic, S. Konjicija, M. Ahic-Djokic and A. Salihbegovic, "Stability issues in discretization of wave equation," 2011 18th International Conference on Systems, Signals and Image Processing, 2011, pp. 1-4.

[13] A. Mukhopadhyay and B. Sheldon, "Deformation and stress in electrode materials for Li-ion batteries", Progress in Materials Science, vol. 63, pp. 58-116, 2014. Available: 10.1016/j.pmatsci.2014.02.001 [Accessed 8 October 2021]. 